\title{
ROTÍFEROS DE LA COMUNIDAD DE MADRID ${ }^{1}$
}

\author{
J. L. Velasco*
}

\begin{abstract}
RESUMEN
En la Comunidad de Madrid han sido citados hasta la fecha 135 taxa de Rotíferos (Rotifera) que representan, aproximadamente, un tercio $(30,6 \%)$ de los 441 taxa descritos en España. En este trabajo se presentan las localidades precisas y los datos de colecta de las especies de Madrid. De las localidades muestreadas destaca por su interés la Laguna de San Juan. De esta laguna, localizada en terrenos yesíferos, se aportan las primeras citas para España de seis species.
\end{abstract}

Palabras clave: Rotíferos, Madrid, España.

\section{ABSTRACT}

\section{Rotifers of Comunidad de Madrid}

The 135 taxa of Rotifers (Rotatoria) reported so far from Comunidad de Madrid, represented almost one third $(30,6 \%)$ of the 441 taxa reported for Spain as a whole. In this paper we present the precise localities and collection data for species located in Madrid. Of all localities sampled, the Laguna de San Juan appears as particularly interesting, since first reports for Spain of six species have been made from this small lake located on gypsum soils.

Key words: Rotifers, Madrid, Spain.

\section{Introducción}

Los rotíferos, metazoos invertebrados de tamaño microscópico -entre 40 y $2.000 \mu \mathrm{m}$ - viven fundamentalmente en aguas dulces, aunque algunos géneros también se encuentran en aguas salobres y atalasohalinas y sólo unas 50 especies son exclusivamente marinas. El número de especies conocido hasta la fecha, unas 2.000 aproximadamente, puede considerarse relativamente pequeño si se compara con otros grupos de invertebrados.
En la Comunidad de Madrid han sido citados hasta la fecha 135 taxa que representan, aproximadamente, un tercio $(30,6 \%)$ de los 441 taxa descritos en España y de los cuales 349 figuran en la Lista faunística y bibliográfica de los Rotiferos (Rotatoria) de la Península Ibérica e Islas Baleares y Canarias (Velasco, 1990).

De estos 135 taxa 10 son nuevas citas para España, siendo la laguna de San Juan la que aporta un mayor número de ellas (seis):

\footnotetext{
* Depto. de Biodiversidad y Biología Evolutiva, Museo Nacional de Ciencias Naturales (CSIC), José Gutiérrez Abascal, 2; E-
} 27006 Madrid. 
Laguna de San Juan:

Notommata glyphura

Colurella uncinata f. deflexa

Cephalodella hoodi

Asplanchna sieboldi

Testudinella patina $\mathrm{f}$. intermedia

Testudinella patina f. trilobata

Parque Regional de la Cuenca Alta del Manzanares:

Trichocerca insulana

Dicranophorus uncinatus

Keratella tropica f. nova

Laguna de El Campillo: Lecane clara

Laguna de Ontígola:

Cephalodella misgurnus

Los primeros rotíferos citados en España, por Forti (1906) y Madrid (1907), fueron encontrados en el estanque del Retiro y en sedimentos del depósito del Canal de Lozoya, ambos medios localizados en Madrid, y corresponden por tanto a esta Comunidad. Keratella quadrata fue la primera especie citada por Forti en 1906, mientras que Epiphanes senta, Rotaria neptunia y Rotaria rotatoria fueron citadas por Madrid un año más tarde.

La Tabla 1 muestra el listado de los 135 rotíferos citados, figurando en la columna de la derecha de dicha tabla los números por los que aparecen ordenadas las referencias bibliográficas que a su vez remiten a los autores y año de dichas citas.

De las dos subclases que componen el Phylum Rotifera, el correspondiente a Bdelloidea está compuesto por organismos que habitan fundamentalmente en suelos húmedos, musgos y líquenes, y algunas especies pueden encontrarse también en el bentos y entre la vegetación acuática de la zona litoral en ecosistemas de agua dulce. La escasa atención prestada a estos organismos en los estudios de fauna edáfica en nuestro país, por una parte, y también la dificultad que presenta su taxonomía, explica que la mayor parte de las citas se deban casi exclusivamente a Donner (1970), destacado especialista de este grupo.

El estudio de los rotíferos de la subclase Monogononta, presentes en la mayor parte de las aguas continentales, ha merecido la atención de numerosos limnólogos debido tanto a la importancia cuantitativa que los representantes de este grupo tienen en el plancton, bentos y zona litoral de ecosistemas acuáticos, como también a su amplia capa- cidad de adaptación a ambientes acuáticos muy variables en cuanto a salinidad, $\mathrm{pH}$, temperatura, nutrientes, contaminantes etc.

El Phylum Rotifera está representado en la Comunidad de Madrid por 5 órdenes, 21 familias, 37 géneros y 135 especies.

Lecane con 17 especies es el género mejor representado, seguido de Trichocerca con 8 especies y Brachionus y Cephalodella con 6.

Una característica común de todos estos géneros es que no son representantes típicos planctónicos, lo cual es explicable teniendo en cuenta que la mayoría de los hábitats muestreados corresponde a medios acuáticos someros. A este respecto, conviene destacar que los medios donde pueden desarrollarse organismos típicamente planctónicos, por las condiciones de profundidad y permanencia de sus aguas, se limitan a dos embalses relativamente grandes -San Juan y El Villar- y pequeños embalses y presas localizados en el Parque Regional de la Cuenca Alta del Manzanares, mientras que la mayoría de las especies citadas pertenecen a las lagunas de San Juan, Ontígola y El Campillo, con zonas litorales bien desarrolladas, y a pequeñas masas de agua -manantiales y charcas de diferentes tipos- ampliamente representadas también en dicho Parque.

La especie más frecuente, citada en 28 medios distintos, es Lecane closterocerca seguida por Lepadella patella, Brachionus quadridentatus y Anuraeopsis fissa, encontradas en 19 medios y Keratella cochlearis (16 medios), Testudinella patina (15 medios), Lecane lunaris y Polyarthra vulgaris (13 medios) y Lecane hamata (11 medios). Así pues, Lecane no es solamente el género con mayor número de especies citadas, sino que además cuenta con la especie más frecuente y otras dos que también figuran entre las encontradas en un mayor número de medios.

\section{AGRADECIMIENTOS}

Este trabajo ha sido subvencionado por el proyecto GR/AMB/0750/2004 de la Comunidad de Madrid.

\section{Referencias}

ArÉvalo, C., 1923. Algunas consideraciones sobre la variación temporal del plancton en aguas de Madrid. Boletín de la Real Sociedad Española de Historia Natural, 23: 94-103. [1]

Arévalo, C., 1929. La vida en las aguas dulces. Colección Labor, Sec. 12 Cienc. Nat., $\mathrm{n}^{\mathrm{o}} 197$. Barcelona. 198 pp. [2] 
Tabla 1.- Relación de las especies de Rotíferos citadas en la Comunidad de Madrid.

Table 1.- Relation of Rotifer species reported in Comunidad de Madrid.

PHYLLUM ROTIFERA

SUBCLASE BDELLOIDEA

\begin{tabular}{|c|c|c|c|c|}
\hline Especie & Localidad & Municipio & UTM & Autor \\
\hline \multicolumn{5}{|l|}{ Orden Adinetida } \\
\hline \multicolumn{5}{|l|}{ Familia Adinetidae } \\
\hline Adineta vaga (Davis 1873) & Vallecas-Vaciamadrid & Madrid & 30 TVK5370 & 3 \\
\hline \multicolumn{5}{|l|}{ Orden Philodina } \\
\hline \multicolumn{5}{|l|}{ Familia Habrotrochidae } \\
\hline Habrotrocha angusticollis (Murray 1905) & Sierra Guadarrama & $?$ & $?$ & 9 \\
\hline H. constricta (Dujardin 1841) & Sierra Guadarrama & Torrelodones & 30TVK2293 & 3 \\
\hline H. flaviformis de Koning 1947 & Sierra Guadarrama & Torrelodones & 30 TVK 2293 & 3 \\
\hline H. pusilla (Bryce 1893$)$ & Sierra Guadarrama & Torrelodones & 30TVK2293 & 3 \\
\hline H. tranquilla Milne 1916 & Sierra Guadarrama & Torrelodones & 30 TVK 2293 & 3 \\
\hline Scephanotrocha simples de Koning 1947 & Vallecas-Vaciamadrid & Madrid & $30 \mathrm{TVK} 5370$ & 3 \\
\hline \multicolumn{5}{|l|}{ Familia Philodinida } \\
\hline \multirow[t]{2}{*}{ Ceratotrocha cornigera (Bryce 1893) } & Vallecas-Vaciamadrid & Madrid & 30 TVK5370 & 3 \\
\hline & Villalva & Villalva & 30TVL1701 & 3 \\
\hline Macrotrachela ehrenbergii (Janson 1893) & Vallecas-Vaciamadrid & Madrid & 30 TVK5370 & 3 \\
\hline M. habita ((Bryce 1894) & Sierra Guadarrama & Torrelodones & 30 TVK 2293 & 3 \\
\hline M. multispinosa brevispina (Murria 1908) & Sierra Guadarrama & Torrelodones & $30 \mathrm{TVK} 2293$ & 3 \\
\hline \multirow[t]{2}{*}{ M. nana (Bryce 1912) } & Sierra Guadarrama & Torrelodones & 30 TVK 2293 & 3 \\
\hline & Sierra Guadarrama & Villalva & 30TVL170 & 3 \\
\hline M. papillosa (Thompson 1892) & Sierra Guadarrama & Torrelodones & 30 TVK 2293 & 3 \\
\hline M. quadricornifera loricata Donner 1965) & Sierra Guadarrama & Torrelodones & 30 TVK 2293 & 3 \\
\hline Mniobia magna (Plate 1889) & Sierra Guadarrama & Torrelodones & 30 TVK 2293 & 3 \\
\hline Philodina flaviceps Bryce 1906 & Sierra Guadarrama & Torrelodones & 30 TVK 2293 & 3 \\
\hline Ph. roseola Ehrenberg 1832 & Estanque Retiro & Madrid & 30TVK4372 & 8 \\
\hline \multirow{2}{*}{ Rotaria neptunia (Ehrenberg 1832) } & Depósito canal Lozoya & Madrid & 30TVK4379 & 6 \\
\hline & Laguna de San Juan & Chinchón & 30TVK2069 & 13 \\
\hline \multirow[t]{4}{*}{ R. rotatoria (Pallas 1776$)$} & Depósito canal Lozoya & Madrid & 30TVK4379 & 6 \\
\hline & Embalse del Villar & Mangirón & 30TVL5334 & 7 \\
\hline & Estanque del Retiro & Madrid & 30TVK4372 & 8 \\
\hline & Laguna de San Juan & Chinchón & 30TVK2069 & 13 \\
\hline \multirow[t]{2}{*}{ R. sordida (Western 1893) } & Sierra Guadarrama & Torrelodones & $30 \mathrm{TVK} 2293$ & 3 \\
\hline & Sierra Guadarrama & Villalva & 30TVL1701 & 3 \\
\hline
\end{tabular}

SUBCLASE MONOGONONTA

\section{Orden Collothecacea}

Familia Collothecidae

Collotheca ornata (Ehrenberg 1832)

Laguna San Juan

Chinchón

30TVK2069

13

Orden Flosculariacea

Familia Testudinellidae

Pompholix sulcata Hudson 1885

Laguna del Campillo

Presa arroyo El Garzo

Laguna del Campillo

Presa arroyo Boquillón

Valtravieso

Laguna de San Juan

Cerca del Indiano

Cantera Jarosa

Monte Egido 6

Canto del Berrueco 1

Canto del Pico

Valtravieso

Cerca de Ramos

Presa arroyo El Garzo

Manantial Cerca de la Venta
Arganda del Rey

Las Rozas

Arganda del Rey

Las Rozas

Colmenar Viejo

Chinchón

Soto del Real

Manzanares el Real

Hoyo de Manzanares

Manzanares el Real

Hoyo de Manzanares

Colmenar Viejo

Colmenar Viejo

Las Rozas

Manzanares el Real
30TVK5964 14

30TVK2788 17

30TVK5964 14

30TVK2689 17

30TVK3195 17

30TVK2069 13

30TVL3113 17

30TVL2609 17

30TVK2599 17

30TVL3013 17

30TVK2295 17

30TVK3195 17

30TVK3498 17

30TVK2788 17

30TVL3105 17 


\begin{tabular}{|c|c|c|c|c|}
\hline Especie & Localidad & Municipio & UTM & Autor \\
\hline \multirow[t]{3}{*}{ T. patina intermedia Anderson 1889} & Laguna de San Juan & Chinchón & 30TVK2069 & 13 \\
\hline & Laguna del Campillo & Arganda del Rey & 30TVK5964 & 14 \\
\hline & Cerca del Indiano & Soto del Real & 30TVL3113 & 17 \\
\hline \multirow[t]{2}{*}{ T. patina triloba Anderson \& Shephard 1892} & Laguna de San Juan & Chinchón & 30 TVK 2069 & 13 \\
\hline & Laguna del Campillo & Arganda del Rey & 30TVK5964 & 14 \\
\hline \multicolumn{5}{|l|}{ Familia Trochosphaeridae } \\
\hline Filinia hofmanni Koste 1980 & Laguna las Madres & Arganda del Rey & 30 TVK5662 & 16 \\
\hline \multirow[t]{8}{*}{ F. longiseta (Ehrenberg 1834) } & $?$ & Aranjuez & 30 TVK 4833 & 10 \\
\hline & La Berzosa 1 & Hoyo de Manzanares & 30TVK 2197 & 17 \\
\hline & La Berzosa 2 & Hoyo de Manzanares & 30 TVK 2197 & 17 \\
\hline & Barranco del Cura & Hoyo de Manzanares & 30TVK2295 & 17 \\
\hline & Cerca de Ramos & Colmenar Viejo & 30 TVK 3498 & 17 \\
\hline & Manantial Lanchar de Castilla & Hoyo de Manzanares & 30TVK2197 & 17 \\
\hline & Fuente de los Pozuelos & Manzanares el Real & 30 TVK 2405 & 17 \\
\hline & Embalse Los Peñascales & Torrelodones & $30 \mathrm{TVK} 2492$ & 17 \\
\hline F. terminalis (Plate 1886) & Laguna de San Juan & Chinchón & 30TVK2069 & 13 \\
\hline \multicolumn{5}{|l|}{ Familia Hexarthridae } \\
\hline \multirow[t]{9}{*}{ Hexarthra mira (Hudson 1871) } & Laguna las Madres & Arganda del Rey & 30TVK5662 & 16 \\
\hline & Presa Cabeza del Lobo & Colmenar Viejo & 30TVK3299 & 17 \\
\hline & Presa arroyo Boquillón & Las Rozas & 30TVK2689 & 17 \\
\hline & Monte Egido 6 & Hoyo de Manzanares & 30 TVK 2599 & 17 \\
\hline & Zaburdón & Cerceda & 30TVL3003 & 17 \\
\hline & Valtravieso & Colmenar Viejo & 30 TVK3195 & 17 \\
\hline & La Berzosa 1 & Hoyo de Manzanares & 30 TVK 2197 & 17 \\
\hline & La Berzosa 2 & Hoyo de Manzanares & 30TVK2197 & 17 \\
\hline & Barranco del Cura & Hoyo de Manzanares & 30 TVK 2295 & 17 \\
\hline H. oxyuris (Sernov 1903) & Laguna de las Madres & Arganda del Rey & 30 TVK5662 & 16 \\
\hline
\end{tabular}

\section{Orden Ploima}

Familia Asplanchnidae

Asplanchna brightwelli Gosse 1650

A. girodi de Guerne 1886

A. priodonta Gosse 1850

A. sieboldi (Leydig 1854)

Familia Brachionidae

Anuraeopsis fissa Gosse 1851

Brachionus angularis Gosse 1851

B. bidentata f. inermis (Rousselet 1906)

B. calyciflorus calyciflorus Pallas 1776

Chinchón

Hoyo de Manzanares

Cerceda

Colmenar Viejo

Las Rozas

Soto del Real

Becerril de la Sierra

Chinchón

Chinchón

Arganda del Rey

Colmenar Viejo

Hoyo de Manzanares

Manzanares el Real

Manzanares el Real

Becerril de la Sierra

Colmenar Viejo

Colmenar Viejo

Manzanares el Real

Hoyo de Manzanares

Hoyo de Manzanares

Manzanares el Real

Manzanares el Real

Colmenar Viejo

Hoyo de Manzanares

Hoyo de Manzanares

Hoyo de Manzanares

Hoyo de Manzanares

Chinchón

Chinchón

Hoyo de Manzanares

Hoyo de Manzanares

Chinchón

Chinchón

Chinchón

Chinchón
30TVK2069 $\quad 13$

30TVK2197 17

30TVL3003 17

30TVK3097 17

30TVK2788 17

30TVL3114 17

30TVL1811 17

30TVK2069 13

30TVK4931 15

30TVK5662 16

30TVK2899 17

30TVK2197 17

30TVL3006 17

30TVL2405 17

30TVL1811 17

30TVK3299 17

30TVK3097 17

30TVL2609 17

30TVK2599 17

30TVK2599 17

30TVL3013 17

30TVL3004 17

30TVK3195 17

30TVK2197 17

30TVK2295 17

30TVK2197 17

30TVK2295 17

30TVK2069 13

30TVK4931 5

30TVK2197 17

30TVK2197 17

30TVK2069 13

30TVK4931 15

30TVK2069 13

30TVK4931 15 


\begin{tabular}{|c|c|c|c|c|}
\hline Especie & Localidad & Municipio & UTM & Autor \\
\hline \multirow{6}{*}{ B. calyciflorus f. amphiceros (Ehrenberg 1838) } & Laguna de las Madres & Arganda del Rey & 30TVK5662 & 16 \\
\hline & Embalse Los Peñascales & Torrelodones & $30 \mathrm{TVK} 2492$ & 17 \\
\hline & Matachiviles & Hoyo de Manzanares & 30TVL3006 & 17 \\
\hline & Estanque del Retiro & Madrid & $30 \mathrm{TVK} 4375$ & 1 \\
\hline & Canto del Pico 5 & Hoyo de Manzanares & 30 TVK 2295 & 17 \\
\hline & Embalse Los Peñascales & Torrelodones & $30 \mathrm{TVK} 2492$ & 17 \\
\hline B. calyciflorus f. anuraeiformis Brehm 1909 & Laguna de San Juan & Chinchón & 30TVK2069 & 13 \\
\hline \multirow[t]{2}{*}{ B. plicatilis O. F. Müller 1786} & Montarco & $?$ & $?$ & 10 \\
\hline & Laguna de Ontígola & Chinchón & 30TVK4931 & 15 \\
\hline \multirow[t]{3}{*}{ B. quadridentatus quadridentatus Hermann 1783} & Estanque del Retiro & Madrid & 30 TVK 4375 & 1 \\
\hline & Estanque del Retiro & Madrid & $30 \mathrm{TVK} 4375$ & 2 \\
\hline & Zaburdón & Cerceda & 30TVL3003 & 17 \\
\hline B. quadridentatus var. ancylognathus (Schmarda 1859) & Estanque del Retiro & Madrid & $30 \mathrm{TVK} 4375$ & ¿1? \\
\hline \multirow[t]{4}{*}{ B. quadridentatus f. brevispinus (Ehrenberg 1832) } & Laguna de San Juan & Chinchón & 30TVK2069 & 13 \\
\hline & Valtravieso & Colmenar Viejo & 30 TVK 3195 & 17 \\
\hline & La Berzosa 1 & Hoyo de Manzanares & 30 TVK 2197 & 17 \\
\hline & La Berzosa 2 & Hoyo de Manzanares & 30 TVK 2197 & 17 \\
\hline \multirow{7}{*}{ B. quadridentatus var. cluniorbicularis (Skorikov 1894) } & Laguna de San Juan & Chinchón & 30 TVK 2069 & 13 \\
\hline & Laguna de Ontígola & Chinchón & 30TVK4931 & 15 \\
\hline & Embalse Los Peñascales & Torrelodones & 30TVK 2492 & 17 \\
\hline & Presa arroyo El Garzo & Las Rozas & 30TVK2788 & 17 \\
\hline & Fuente del Piojo & Madrid & 30TVK4085 & 17 \\
\hline & La Berzosa 1 & Hoyo de Manzanares & 30 TVK 2197 & 17 \\
\hline & Canto del Pico 5 & Hoyo de Manzanares & 30 TVK 2295 & 17 \\
\hline \multirow[t]{4}{*}{ B. quadridentatus f. rhenanus (Lauterborn 1893) } & Laguna de San Juan & Chinchón & 30TVK2069 & 13 \\
\hline & Laguna de Ontígola & Chinchón & $30 \mathrm{TVK} 4931$ & 15 \\
\hline & Embalse Los Peñascales & Torrelodones & $30 \mathrm{TVK} 2492$ & 17 \\
\hline & La Berzosa 1 & Hoyo de Manzanares & $30 \mathrm{TVK} 2197$ & 17 \\
\hline \multirow[t]{3}{*}{ B. urceolaris urceoloris O. F. Müller 1773} & Laguna de San Juan & Chinchón & 30 TVK 2069 & 13 \\
\hline & Embalse Los Peñascales & Torrelodones & 30 TVK 2492 & 17 \\
\hline & Presa arroyo Boquillón & Las Rozas & 30 TVK 2689 & 17 \\
\hline B. urceolaris var. rubens (Ehrenberg 1838) & Laguna de San Juan & Chinchón & 30TVK2069 & 13 \\
\hline \multirow[t]{16}{*}{ Keratella cochlearis cochlearis (Gosse 1851) } & Estanque del Retiro & Madrid & 30 TVK 4375 & 1 \\
\hline & Estanque del Retiro & Madrid & 30 TVK 4375 & 11 \\
\hline & Embalse de San Juan & S. Martín Valdeiglesias & 30 TUK 8870 & 12 \\
\hline & Laguna del Campillo & Arganda del Rey & 30 TVK5964 & 14 \\
\hline & Laguna de Ontígola & Chinchón & $30 \mathrm{TVK} 4931$ & 15 \\
\hline & Laguna de las Madres & Arganda del Rey & 30 TVK 5662 & 16 \\
\hline & Cantera Jarosa & Manzanares el Real & 30TVL2609 & 17 \\
\hline & Zaburdón & Cerceda & 30TVL3003 & 17 \\
\hline & Canto del Berrueco & Manzanares el Real & 30TVL3013 & 17 \\
\hline & Canto del Pico 1 & Hoyo de Manzanares & 30 TVK 2295 & 17 \\
\hline & La Berzosa 1 & Hoyo de Manzanares & 30 TVK 2197 & 17 \\
\hline & Canto del Pico 5 & Hoyo de Manzanares & 30 TVK 2295 & 17 \\
\hline & Barranco del Cura & Hoyo de Manzanares & 30TVK 2295 & 17 \\
\hline & Matachiviles & Manzanares el Real & 30TVL3006 & 17 \\
\hline & Embalse del Chiquillo & Navacerrada & 30TVL1511 & 17 \\
\hline & Presa arroyo El Garzo & Las Rozas & 30 TVK 2788 & 17 \\
\hline \multirow[t]{6}{*}{ K. cochlearis var. tecta Gosse 1886} & Presa de La Barranca 2 & Navacerrada & 30TVL1612 & 17 \\
\hline & Embalse del Chiquillo & Navacerrada & 30TVL1511 & 17 \\
\hline & Embalse de Majalespino & Becerril de la Sierra & 30TVL1811 & 17 \\
\hline & Embalse de Los Ciervos & Colmenar Viejo & 30TVK3097 & 17 \\
\hline & Presa arroyo El Garzo & Las Rozas & 30 TVK 2788 & 17 \\
\hline & Zaburdón & Cerceda & 30TVL3003 & 17 \\
\hline \multirow[t]{2}{*}{ K. procurva (Torpe 1891) } & Charca artificial en Centro F. & & & \\
\hline & González Bernáldez & Soto del Real & 30TVL3412 & 17 \\
\hline \multirow[t]{8}{*}{ K. quadrata (O. F. Müller 1786) } & Estanque del Retiro & Madrid & $30 \mathrm{TVK} 4375$ & 1 \\
\hline & Estanque del Retiro & Madrid & 30 TVK4375 & 4 \\
\hline & Estanque del Retiro & Madrid & 30 TVK4375 & 8 \\
\hline & Aranjuez & Aranjuez & $30 \mathrm{TVK} 4833$ & 10 \\
\hline & Estanque del Retiro & Madrid & 30 TVK4375 & 11 \\
\hline & Laguna de San Juan & Chinchón & 30TVK2069 & 13 \\
\hline & Laguna de las Madres & Arganda del Rey & 30 TVK 5662 & 16 \\
\hline & Monte Egido 6 & Hoyo de Manzanares & 30 TVK 2599 & 17 \\
\hline \multirow[t]{2}{*}{ K. tropica tropica Apstein 1907} & Laguna de San Juan & Chinchón & 30 TVK 2069 & 13 \\
\hline & Laguna de Ontígola & Chinchón & 30TVK4931 & 15 \\
\hline K. tropica f. nova & Barranco del Cura & Hoyo de Manzanare & 30TVK2295 & 17 \\
\hline
\end{tabular}




\begin{tabular}{|c|c|c|c|c|}
\hline Especie & Localidad & Municipio & UTM & Autor \\
\hline K. valga (Ehrenberg 1834) & Estanque del Retiro & Madrid & 30TVK4375 & 1 \\
\hline \multirow{2}{*}{ Notholca acuminata (Ehrenberg 1832) } & Laguna de San Juan & Chinchón & 30 TVK 2069 & 13 \\
\hline & Laguna de Ontígola & Chinchón & 30TVK4931 & 15 \\
\hline N. squamula (O. F. Müller 1786) & Laguna de las Madres & Arganda del Rey & 30 TVK 5662 & 16 \\
\hline \multirow[t]{3}{*}{ Platyias quadricornis (Ehrenberg 1832) } & Laguna de las Madres & Arganda del Rey & 30 TVK5662 & 16 \\
\hline & Cantera Jarosa & Manzanares el Real & 30TVL2609 & 17 \\
\hline & Manantial Cerca de la Venta & Manzanares el Real & 30TVL3105 & 17 \\
\hline \multicolumn{5}{|l|}{ Familia Dicranophoridae } \\
\hline \multirow[t]{2}{*}{ Dicranophorus epicharis Harring \& Myers 1928} & Canto del Pico 5 & Hoyo de Manzanares & 30 TVK 2295 & 17 \\
\hline & Monte Egido & Hoyo de Manzanares & 30 TVK 2599 & 17 \\
\hline D. forcipatus (O. F. Müller 1786) & Laguna de San Juan & Chinchón & 30 TVK 2069 & 13 \\
\hline D. uncinatus (Milne 1886) & Manantial Cerca de la Venta & Manzanares el Real & 30TVL3105 & 17 \\
\hline \multirow[t]{2}{*}{ Encentrum mustela (Milne 1885) } & Vallecas-Vaciamadrid & Madrid & 30 TVK 5370 & 3 \\
\hline & Laguna de San Juan & Chinchón & 30TVK2069 & 13 \\
\hline E. (p) plicatum (Eyferth 1878) & Laguna de Ontígola & Chinchón & 30TVK4931 & 15 \\
\hline \multicolumn{5}{|l|}{ Familia Epiphanidae } \\
\hline Epiphanes senta (O. F. Müller 1773) & Canal de Lozoya & Madrid & $30 \mathrm{TVK} 4379$ & 6 \\
\hline \multicolumn{5}{|l|}{ Familia Euchlanidae } \\
\hline \multirow[t]{2}{*}{ Euchlanis deflexa (Gosse 1851) } & Laguna del Campillo & Arganda del Rey & 30TVK5964 & 14 \\
\hline & Valtravieso & Colmenar Viejo & 30TVK3097 & 17 \\
\hline \multirow[t]{4}{*}{ E. dilatata Ehrenberg 1832} & Manantial Huertos del Pajarejo & Colmenar Viejo & 30 TVK 2899 & 17 \\
\hline & La Berzosa 2 & Hoyo de Manzanares & 30TVK 2197 & 17 \\
\hline & Presa Cabeza del Lobo & Colmenar Viejo & 30TVK3299 & 17 \\
\hline & Presa arroyo de El Garzo & Las Rozas & $30 \mathrm{TVK} 2788$ & 17 \\
\hline \multicolumn{5}{|l|}{ Familia Gastropodidae } \\
\hline \multirow[t]{3}{*}{ Ascomorpha saltans Bartsch 1879} & Laguna de las Madres & Arganda del Rey & 30 TVK5662 & 16 \\
\hline & Canto del Berrueco & Manzanares el Real & 30TVL3013 & 17 \\
\hline & Barranco del Cura & Hoyo de Manzanares & 30 TVK 2295 & 17 \\
\hline \multicolumn{5}{|l|}{ Familia Lecanidae } \\
\hline Lecane aculeata (jakubski 1912) & Laguna de las Madres & Arganda del Rey & 30 TVK5662 & 16 \\
\hline \multirow[t]{3}{*}{ L. arcuata (Bryce 1891) } & Laguna de San Juan & Chinchón & 30TVK 2069 & 13 \\
\hline & Laguna del Campillo & Arganda del Rey & 30TVK5964 & 14 \\
\hline & Laguna de Ontígola & Chinchón & 30TVK4931 & 15 \\
\hline \multirow[t]{7}{*}{ L. bulla (Gosse 1851) } & Laguna de las Madres & Arganda del Rey & 30TVK5662 & 16 \\
\hline & Canto del Berrueco 1 & Manzanares el Real & 30TVL3013 & 17 \\
\hline & Canto del Pico 5 & Hoyo de Manzanares & 30TVK 2295 & 17 \\
\hline & Las Zahúrdas 1 & Las Rozas & 30TVK 2687 & 17 \\
\hline & Presa Cabeza del Lobo & Colmenar Viejo & 30TVK3299 & 17 \\
\hline & Manantial Cerca de la Venta & Manzanares el Real & 30TVL3105 & 17 \\
\hline & Fuente de Los Pozuelos & Manzanares el Real & 30TVL2405 & 17 \\
\hline L. clara $($ Bryce 1892$)$ & Laguna del Campillo & Arganda del Rey & 30TVK5964 & 14 \\
\hline L. closterocerca (Schmarda 1859) & Laguna de San Juan & Chinchón & 30TVK5964 & 13 \\
\hline & Laguna del Campillo & Arganda del Rey & 30TVK4931 & 14 \\
\hline & Laguna de Ontígola & Chinchón & 30TVK5662 & 15 \\
\hline & Laguna de las Madres & Arganda del Rey & 30TVK5662 & 16 \\
\hline & Cerca del Indiano & Soto del Real & 30TVL3113 & 17 \\
\hline & Zaburdón & Cerceda & 30TVL3003 & 17 \\
\hline & Canto del Berrueco 1 & Manzanares el Real & 30TVL3013 & 17 \\
\hline & Charca en carretera M-607 & Manzanares el Real & 30TVL3004 & 17 \\
\hline & Canto del Pico 1 & Hoyo de Manzanares & 30TVK 2295 & 17 \\
\hline & Canto del Pico 5 & Hoyo de Manzanares & 30TVK 2295 & 17 \\
\hline & La Berzosa 2 & Hoyo de Manzanares & 30TVK 2197 & 17 \\
\hline & Barranco del Cura & Hoyo de Manzanares & 30 TVK 2295 & 17 \\
\hline & Cerca de Ramos & Colmenar Viejo & 30TVK3498 & 17 \\
\hline & Las Zahúrdas 1 & Las Rozas & 30 TVK 2687 & 17 \\
\hline & Presa de La Barranca 2 & Navacerrada & 30TVL1612 & 17 \\
\hline & Presa Cabeza del Lobo & Colmenar Viejo & 30TVK3299 & 17 \\
\hline & Embalse de Los Ciervos & Colmenar Viejo & 30TVK3097 & 17 \\
\hline & Presa arroyo de El Garzo & Las Rozas & 30TVK 2788 & 17 \\
\hline & Presa arroyo Boquillón & Las Rozas & $30 \mathrm{TVK} 2689$ & 17 \\
\hline & Manantial Huertos del Pajarejo & Colmenar Viejo & 30 TVK 2899 & 17 \\
\hline & Presa de la Barranca 2 & & 30TVL1312 & 17 \\
\hline & Manantial de Cañacerral & Colmenar Viejo & 30 TVK3096 & 17 \\
\hline & Manantial Cerca de la Venta & Manzanares el Real & 30TVL3105 & 17 \\
\hline & Matachiviles & Manzanares el Real & 30TVL3006 & 17 \\
\hline
\end{tabular}




\begin{tabular}{|c|c|c|c|c|}
\hline Especie & Localidad & Municipio & UTM & Autor \\
\hline & Embalse del Chiquillo & Navacerrada & 30TVL1511 & 17 \\
\hline & Fuente de Las Cochiqueras & S. Sebastián de los Reyes & 30TVK4493 & 17 \\
\hline & Fuente del Piojo & Madrid & $30 \mathrm{TVK} 4085$ & 17 \\
\hline & Fuente de San Isidro & S. Sebastián de los Reyes & 30TVK5095 & 17 \\
\hline \multirow{6}{*}{ L. flexilis (Gosse 1886) } & Laguna de San Juan & Chinchón & 30 TVK2069 & 13 \\
\hline & Laguna las Madres & Arganda del Rey & 30 TVK5662 & 16 \\
\hline & Manantial Cerca de la Venta & Manzanares el Real & 30TVL3105 & 17 \\
\hline & Matachiviles & Manzanares el Real & 30TVL3006 & 17 \\
\hline & Fuente de Los Pozuelos & Manzanares el Real & 30TVL2405 & 17 \\
\hline & Canto del Berrueco 1 & Manzanares el Real & $30 \mathrm{VL} 3013 \mathrm{~T}$ & 17 \\
\hline L. furcata (Murria 1913) & Canto del Berrueco 1 & Manzanares el Real & 30 VL3013T & 17 \\
\hline \multirow[t]{11}{*}{ L. hamata (Stokes 1896) } & Laguna de San Juan & Chinchón & $30 \mathrm{TVK} 2069$ & 13 \\
\hline & Laguna las Madres & Arganda del Rey & 30 TVK5662 & 16 \\
\hline & Manantial Cerca de la Venta & Manzanares el Real & 30TVL3105 & 17 \\
\hline & Fuente de Los Pozuelos & Manzanares el Real & 30TVL2405 & 17 \\
\hline & Cerca del Indiano & Soto del Real & 30TVL3113 & 17 \\
\hline & Zaburdón & Cerceda & 30TVL3003 & 17 \\
\hline & Canto del Berrueco 1 & Manzanares el Real & 30TVL3013 & 17 \\
\hline & Canto del Pico 1 & Hoyo de Manzanares & 30 TVK 2295 & 17 \\
\hline & Canto del Pico 5 & Hoyo de Manzanares & 30TVK 2295 & 17 \\
\hline & Cerca de Ramos & Colmenar Viejo & 30TVK 3498 & 17 \\
\hline & Presa arroyo de El Garzo & Las Rozas & 30 TVK 2788 & 17 \\
\hline L. inermis (Bryce 1892) & Sierra de Guadarrama & Torrelodones & 30 TVK2293 & 3 \\
\hline \multirow[t]{7}{*}{ L. luna (O. F. Müller 1776) } & Estanque del Retiro & Madrid & 30 TVK4375 & 1 \\
\hline & Laguna de San Juan & Chinchón & 30TVK2069 & 13 \\
\hline & Laguna del Campillo & Arganda del Rey & 30TVK5964 & 14 \\
\hline & Laguna las Madres & Arganda del Rey & 30 TVK5662 & 16 \\
\hline & Cantera Jarosa & Manzanares el Real & 30TVL2609 & 17 \\
\hline & La Berzosa 1 & Hoyo de Manzanares & 30TVK 2197 & 17 \\
\hline & Barranco Del Cura & Hoyo de Manzanares & 30TVK 2295 & 17 \\
\hline \multirow[t]{10}{*}{ L. lunaris lunaris (Ehrenberg 1832) } & Laguna de San Juan & Chinchón & 30 TVK 2069 & 13 \\
\hline & Laguna del Campillo & Arganda del Rey & 30TVK5964 & 14 \\
\hline & Laguna de Ontígola & Chinchón & 30TVK4931 & 15 \\
\hline & Laguna las Madres & Arganda del Rey & 30 TVK 5662 & 16 \\
\hline & Cerca del Indiano & Soto del Real & 30TVL3113 & 17 \\
\hline & Charca en carretera M-607 & Manzanares el Real & 30TVL3004 & 17 \\
\hline & Barranco Del Cura & Manzanares el Real & 30TVK 2295 & 17 \\
\hline & Manantial Lanchar de Castilla & Hoyo de Manzanares & $30 \mathrm{TVK} 2197$ & 17 \\
\hline & Matachiviles & Manzanares el Real & 30TVL3006 & 17 \\
\hline & Fuente de Los Pozuelos & Manzanares el Real & 30TVL2405 & 17 \\
\hline \multirow[t]{3}{*}{ L. lunaris var. constricta (Murray 1913) } & Laguna de San Juan & Chinchón & 30TVK2069 & 13 \\
\hline & Laguna del Campillo & Arganda del Rey & 30TVK5964 & 14 \\
\hline & Laguna las Madres & Arganda del Rey & 30TVK5662 & 16 \\
\hline \multirow[t]{2}{*}{ L. mira (Murray 1913) } & Laguna las Madres & Arganda del Rey & 30TVK5662 & 16 \\
\hline & Cerca del Indiano & Soto del Real & 30TVL3113 & 17 \\
\hline \multirow[t]{2}{*}{ L. nana (Murray 1913) } & Canto del Pico 5 & Hoyo de Manzanares & 30TVK 2295 & 17 \\
\hline & Barranco Del Cura & Manzanares el Real & 30 TVK 2295 & 17 \\
\hline L. obtusa (Murray 1913) & Laguna las Madres & Arganda del Rey & 30TVK5662 & 16 \\
\hline L. pyriformis (Daday 1905) & Laguna de San Juan & Chinchón & 30TVK2069 & 13 \\
\hline L. stichaea (Harring 1913) & La Berzosa 2 & Hoyo de Manzanare & 30 TVK 2197 & 17 \\
\hline L. subtilis Harring \& Myers 1926 & Laguna las Madres & Arganda del Rey & 30 TVK5662 & 16 \\
\hline \multicolumn{5}{|l|}{ Familia Lepadellidae } \\
\hline \multirow[t]{3}{*}{ Colurella adriatica Ehrenberg 1831} & Laguna de San Juan & Chinchón & 30TVK5964 & 13 \\
\hline & Laguna del Campillo & Arganda del Rey & 30TVK4931 & 14 \\
\hline & Laguna de Ontígola & Chinchón & 30TVK5662 & 15 \\
\hline \multirow[t]{4}{*}{ C. colurus (Ehrenberg 1830) } & Laguna de San Juan & Chinchón & 30TVK5964 & 13 \\
\hline & Laguna del Campillo & Arganda del Rey & 30TVK4931 & 14 \\
\hline & Laguna de Ontígola & Chinchón & 30 TVK 5662 & 15 \\
\hline & Laguna las Madres & Arganda del Rey & 30 TVK 5662 & 16 \\
\hline \multirow[t]{2}{*}{ C. obtusa (Gosse 1886) } & Laguna de San Juan & Chinchón & 30 TVK2069 & 13 \\
\hline & Laguna de Ontígola & Chinchón & 30TVK4931 & 15 \\
\hline \multirow[t]{2}{*}{ C. uncinata (O. F. Müller 1773) } & Zaburdón & Cerceda & 30TVL3003 & 17 \\
\hline & Canto del Berrueco 1 & Manzanares el Real & 30TVL3013 & 17 \\
\hline \multirow[t]{2}{*}{ C. uncinata f. deflexa (Ehrenberg 1834) } & Laguna de San Juan & Chinchón & 30TVK2069 & 13 \\
\hline & Fuente de Las Cochiqueras & S. Sebastián de los Reyes & 30 TVK4493 & 17 \\
\hline
\end{tabular}




\begin{tabular}{|c|c|c|c|c|}
\hline Especie & Localidad & Municipio & UTM & Autor \\
\hline \multirow[t]{3}{*}{ C. uncinata f. bicuspidata (Ehrenberg 1832) } & Cerca del Indiano & Soto del Real & 30 TVL3113 & 17 \\
\hline & Charca carretera M-607 & Manzanares el Real & 30TVL3004 & 17 \\
\hline & Barranco del Cura & Hoyo de Manzanares & 30 TVK 2295 & 17 \\
\hline \multirow[t]{2}{*}{ Squatinella mutica (Ehrenberg 1832) } & Laguna de Ontígola & Aranjuez & 30TVK4931 & 15 \\
\hline & Canto del Berrueco 1 & Manzanares el Real & 30TVL3013 & 17 \\
\hline \multirow[t]{3}{*}{ Lepadella acuminata (Ehrenberg 1834) } & Sierra de Guadarrama & $?$ & $?$ & 9 \\
\hline & Presa de La Barranca 2 & Navacerrada & 30TVL1612 & 17 \\
\hline & Matachiviles & Manzanares el Real & 30TVL3006 & 17 \\
\hline L. ovalis (O. F. Müller 1786) & Casa de Campo & Madrid & 30 TVK 3875 & 10 \\
\hline L. patella patella (O. F. Müller 1786) & Monte Egido 7 & Hoyo de Manzanares & 30 TVK 2599 & 17 \\
\hline \multirow[t]{14}{*}{ L. patella f. similis (Luks 1912) } & Laguna de San Juan & Chinchón & $30 \mathrm{TVK} 2069$ & 13 \\
\hline & Laguna de Ontígola & Chinchón & 30TVK4931 & 15 \\
\hline & Presa de La Barranca 2 & Navacerrada & 30TVL1612 & 17 \\
\hline & Manantial de Cañacerral & Colmenar Viejo & 30TVK3096 & 17 \\
\hline & Fuente de Los Pozuelos & Manzanares el Real & 30TVL2405 & 17 \\
\hline & Fuente de Las Cochiqueras & S. Sebastián de los Reyes & 30TVK4493 & 17 \\
\hline & Cerca del Indiano & Soto del Real & 30TVL3113 & 17 \\
\hline & Monte Egido 6 & Hoyo de Manzanares & 30 TVK 2599 & 17 \\
\hline & Zaburdón & Cerceda & 30TVL3003 & 17 \\
\hline & Canto del Berrueco 1 & Manzanares el Real & 30TVL3013 & 17 \\
\hline & Charca carretera M-607 & Manzanares el Real & 30TVL3004 & 17 \\
\hline & Canto del Pico 1 & Hoyo de Manzanares & 30TVK 2295 & 17 \\
\hline & Canto del Pico 5 & Hoyo de Manzanares & 30TVK 2295 & 17 \\
\hline & Cerca de Ramos & Colmenar Viejo & 30TVK3498 & 17 \\
\hline \multirow[t]{4}{*}{ L. patella f. oblonga (Ehrenberg 1834) } & Embalse de Los Ciervos & Colmenar Viejo & 30TVK3097 & 17 \\
\hline & Presa arroyo El Garzo & Las Rozas & 30TVK 2788 & 17 \\
\hline & Manantial Cerca de la Venta & Manzanares el Real & 30TVL3105 & 17 \\
\hline & Barranco del Cura & Hoyo de Manzanares & 30 TVK 2295 & 17 \\
\hline L. romboides (Gosse 1886) & Presa arroyo El Garzo & Las Rozas & $30 \mathrm{TVK} 2788$ & 17 \\
\hline L. triptera (Ehrenberg 1830) & Canto del Berrueco 1 & Manzanares el Real & 30TVL3013 & 17 \\
\hline \multicolumn{5}{|l|}{ Familia Lindiidae } \\
\hline Lindia torulosa Dujardin 1841 & Matachiviles & Manzanares el Real & 30TVL3006 & 17 \\
\hline \multicolumn{5}{|l|}{ Familia Mytilinidae } \\
\hline \multirow[t]{2}{*}{ Lophocharis oxysternon (Gosse 1851) } & Laguna de San Juan & Chinchón & 30TVK2069 & 13 \\
\hline & Laguna de Ontígola & Chinchón & $30 \mathrm{TVK} 4931$ & 15 \\
\hline \multirow[t]{2}{*}{ L. salpina (Ehrenberg 1834) } & Laguna las Madres & Arganda del Rey & 30 TVK5662 & 16 \\
\hline & Manantial Cerca de la Venta & Manzanares el Real & 30TVL3105 & 17 \\
\hline \multirow[t]{4}{*}{ Mytilina mucronata (O. F. Müller 1773) } & Fuente de San Isidro & S. Sebastián de los Reyes & 30TVK5095 & 17 \\
\hline & Cerca del Indiano & Soto del Real & 30TVL3113 & 17 \\
\hline & Valtravieso & Colmenar Viejo & 30TVK3195 & 17 \\
\hline & La Berzosa 2 & Hoyo de Manzanares & 30TVK 2197 & 17 \\
\hline \multirow[t]{4}{*}{ M. ventralis var. brevispina Ehrenberg 1932} & Presa Cabeza del Lobo & Colmenar Viejo & 30TVK3299 & 17 \\
\hline & Embalse de Los Ciervos & Colmenar Viejo & 30TVK3097 & 17 \\
\hline & Charca carretera M-607 & Manzanares el Real & 30TVL3004 & 17 \\
\hline & Canto del Pico 5 & Hoyo de Manzanares & 30TVK2295 & 17 \\
\hline \multicolumn{5}{|l|}{ Familia Notommatidae } \\
\hline Cephalodella catellina O. F. Müller 1786 & Laguna de San Juan & Chinchón & 30TVK2069 & 13 \\
\hline C. delicata Wulfert 1937 & Laguna de Ontígola & Chinchón & 30TVK4931 & 15 \\
\hline C. forficula (Ehrenberg 1832) & Laguna de San Juan & Chinchón & 30TVK2069 & 13 \\
\hline C. gibba (Ehrenberg 1832) & Zaburdón & Cerceda & 30TVL3003 & 17 \\
\hline \multirow[t]{2}{*}{ C. gibba microdactyla Koch-Althaus 1963} & Laguna de San Juan & Chinchón & 30TVK2069 & 13 \\
\hline & Laguna del Campillo & Arganda del Rey & 30TVK5964 & 14 \\
\hline C. hoodi (Gosse 1886) & Laguna de San Juan & Chinchón & 30 TVK 2069 & 13 \\
\hline \multirow[t]{2}{*}{ C. misgurnus Wulfert 1937} & Laguna de San Juan & Chinchón & 30TVK2069 & 13 \\
\hline & Laguna las Madres & Arganda del Rey & 30 TVK 5662 & 16 \\
\hline Monommata grandis Tessin 1890 & Laguna las Madres & Arganda del Rey & 30 TVK5662 & 16 \\
\hline M. longiseta (Müller 1786) & Cerca del Indiano & Soto del Real & 30TVL3113 & 17 \\
\hline Notommata glyphura Wulfert 1935 & Laguna de San Juan & Chinchón & 30TVK2069 & 13 \\
\hline Familia Synchaetidae & & & & \\
\hline Polyarthra dolichoptera Idelson 1925 & Laguna de San Juan & Chinchón & 30 TVK 2069 & 13 \\
\hline & Laguna del Campillo & Arganda del Rey & 30 TVK5964 & 14 \\
\hline & Laguna de Ontígola & Chinchón & 30TVK4931 & 15 \\
\hline & Laguna las Madres & Arganda del Rey & 30 TVK5662 & 16 \\
\hline P. euriptera Wiezejski 1891 & Embalse de Los Ciervos & Colmenar Viejo & 30TVK3097 & 17 \\
\hline
\end{tabular}




\begin{tabular}{|c|c|c|c|c|}
\hline Especie & Localidad & Municipio & UTM & Autor \\
\hline \multirow[t]{4}{*}{ P. longiremis Carlin 1943} & Laguna de San Juan & Chinchón & 30TVK2069 & 13 \\
\hline & Embalse de Los Peñascales & Torrelodones & 30TVK 2492 & 17 \\
\hline & Presa arroyo El Garzo & Las Rozas & 30 TVK 2788 & 17 \\
\hline & Presa arroyo Boquillón & Las Rozas & $30 \mathrm{TVK} 2789$ & 17 \\
\hline \multirow[t]{4}{*}{ P. remata Skorikov 1896} & Laguna del Campillo & Arganda del Rey & 30TVK5964 & 14 \\
\hline & Laguna las Madres & Arganda del Rey & 30TVK5662 & 16 \\
\hline & Canto del Pico 1 & Hoyo de Manzanares & 30 TVK 2295 & 17 \\
\hline & La Berzosa 1 & Hoyo de Manzanares & 30 TVK 2197 & 17 \\
\hline \multirow[t]{2}{*}{ P. remata f. proloba (Albertova 1960) } & Canto del Pico 1 & Hoyo de Manzanares & 30 TVK 2295 & 17 \\
\hline & La Berzosa 1 & Hoyo de Manzanares & 30 TVK 2197 & 17 \\
\hline \multirow[t]{13}{*}{ P. vulgaris Carlin 1943} & Estanque del Retiro & Madrid & $30 \mathrm{TVK} 4375$ & 1 \\
\hline & Embalse San Juan & S. Martín Valdeiglesias & 30 TUK 8870 & 12 \\
\hline & Presa de La Barranca 2 & Navacerrada & 30TVL1612 & 17 \\
\hline & Depósito «Los Palancares» & Soto del Real & 30TVL3114 & 17 \\
\hline & Embalse del Chiquillo & Navacerrada & 30TVL1511 & 17 \\
\hline & Embalse de Majalespino & Becerril de la Sierra & 30TVL1811 & 17 \\
\hline & Cantera Jarosa & Manzanares el Real & 30TVL2609 & 17 \\
\hline & Zaburdón & Cerceda & 30TVL3003 & 17 \\
\hline & Monte Egido 7 & Hoyo de Manzanares & 30 TVK 2599 & 17 \\
\hline & Hoyo de Manzanare & Hoyo de Manzanares & 30 TVK 2295 & 17 \\
\hline & La Berzosa 2 & Hoyo de Manzanares & 30 TVK 2197 & 17 \\
\hline & Barranco del Cura & Hoyo de Manzanares & 30 TVK 2295 & 17 \\
\hline & Las Zahúrdas 1 & Las Rozas & 30 TVK 2687 & 17 \\
\hline \multirow[t]{3}{*}{ P. vulgaris f. proloba (Albertova 1960) } & La Berzosa 2 & Hoyo de Manzanares & 30 TVK 2197 & 17 \\
\hline & Zaburdón & Cerceda & 30TVL3003 & 17 \\
\hline & Embalse del Chiquillo & Navacerrada & 30TVL1511 & 17 \\
\hline Synchaeta oblonga Ehrenberg 1831 & Laguna de San Juan & Chinchón & 30 TVK 2069 & 13 \\
\hline \multirow[t]{5}{*}{ S. pectinata Ehrenberg 1832} & Estanque del Retiro & Madrid & 30 TVK 4375 & 1 \\
\hline & Zaburdón & Cerceda & 30TVL3003 & 17 \\
\hline & Barranco del Cura & Hoyo de Manzanares & 30 TVK 2197 & 17 \\
\hline & Las Zahúrdas 1 & Las Rozas & 30 TVK 2687 & 17 \\
\hline & Embalse de Los Ciervos & Colmenar Viejo & 30TVK3097 & 17 \\
\hline \multirow[t]{2}{*}{ S. tremula (O. F. Müller 1786) } & Laguna de San Juan & Chinchón & 30 TVK 2069 & 13 \\
\hline & Laguna de Ontígola & Chinchón & 30TVK4931 & 15 \\
\hline \multicolumn{5}{|l|}{ Familia Trichocercidae } \\
\hline Trichocerca brachyura (Gosse 1851) & Sierra de Guadarrama & $?$ & $?$ & 9 \\
\hline T. cilindrica chatoni (De Beauchamp 1907) & Embalse & $?$ & $?$ & 5 \\
\hline T. elongata (Gosse 1886) & Monte Egido 6 & Hoyo de Manzanare & 30 TVK 2599 & 17 \\
\hline T. insulana (Hauer 1937) & Manantial Huertos del Pajarejo & Colmenar Viejo & 30 TVK2899 & 17 \\
\hline \multirow[t]{5}{*}{ T. longiseta (Schrank 1802) } & Estanque del Retiro & Madrid & 30 TVK4375 & 1 \\
\hline & Embalse San Juan & S. Martín Valdeiglesias & 30TUK 8870 & 12 \\
\hline & Fuente de Los Pozuelos & Manzanares el Real & 30TVL2405 & 17 \\
\hline & Monte Egido 7 & Hoyo de Manzanares & 30 TVK 2599 & 17 \\
\hline & Barranco del Cura & Hoyo de Manzanares & 30 TVK 2295 & 17 \\
\hline \multirow[t]{4}{*}{ T. pusilla (Jennings 1903) } & Matachiviles & Manzanares el Real & 30TVL3006 & 17 \\
\hline & Embalse de Los Ciervos & Colmenar Viejo & 30TVK3097 & 17 \\
\hline & Monte Egido 6 & Hoyo de Manzanares & 30 TVK 2599 & 17 \\
\hline & Charca carretera M-607 & Manzanares el Real & 30TVL3004 & 17 \\
\hline \multirow[t]{3}{*}{ T. rattus (Müller 1786) } & Laguna de San Juan & Chinchón & 30 TVK2069 & 13 \\
\hline & Zaburdón & Cerceda & 30TVL3003 & 17 \\
\hline & La Berzosa 2 & Hoyo de Manzanares & $30 \mathrm{TVK} 2197$ & 17 \\
\hline \multirow[t]{4}{*}{ T. similis (Wierzejski 1893) } & Embalse del Chiquillo & Navacerrada & 30TVL1511 & 17 \\
\hline & Zaburdón & Cerceda & 30TVL3003 & 17 \\
\hline & Canto del Pico 1 & Hoyo de Manzanares & 30 TVK 2295 & 17 \\
\hline & Fuente de Los Pozuelos & Manzanares el Real & 30TVL2405 & 17 \\
\hline T. weberi (Jennings 1903) & Valtravieso & Colmenar Viejo & 30TVK3195 & 17 \\
\hline \multicolumn{5}{|l|}{ Familia Trichotriidae } \\
\hline \multirow[t]{6}{*}{ Trichotria pocillum (O. F. Müller 1776) } & Estanque del Retiro & Madrid & $30 \mathrm{TVK} 4375$ & 1 \\
\hline & Laguna de San Juan & Chinchón & 30 TVK2069 & 13 \\
\hline & Laguna de Ontígola & Chinchón & 30TVK4931 & 15 \\
\hline & Cantera Jarosa & Manzanares el Real & 30TVL2609 & 17 \\
\hline & Zaburdón & Cerceda & 30TVL3003 & 17 \\
\hline & Canto del Pico 5 & Hoyo de Manzanares & 30 TVK 2295 & 17 \\
\hline
\end{tabular}


DONNER, J., 1970. Rotatorien aus einigen Boden und Moosen Spaniens und seiner Insein. Revue d'Écologie et de Biologie du Sol, 7: 501-532. [3]

ForTI, A., 1906. Alcuni appunti sulla composizione del plakton dell «Estanque grande», nel parco dell "Buen Retiro», in Madrid. Módena. [4]

Guiset, A. 1976. Rotíferos. In: Margalef, R. et al. Limnología de los embalses españoles. Dirección General de Hobras Hidráulicas (MOPU). Madrid: 204-236. [5]

MADRID, J., 1907. Análisis micrográficos de los sedimentos del depósito del Canal de Lozoya. Boletín de la Real Sociedad Española de Historia Natural (Notas y Comunicaciones), 7: 393-396. [6]

MADRID, J., 1911a. Datos para el estudio del plancton del río Lozoya. Boletín de la Real Sociedad Española de Historia Natural (Notas y Comunicaciones), 11: 173 176. [7]

MADRID, J., 1911b. El plancton del Estanque grande del Retiro. Boletín de la Real Sociedad Española de Historia Natural (Notas y Comunicaciones), 11: 277 288. [8]

Margalef, R., 1949. Datos para la hidrobiología de la Sierra de Guadarrama. Publicaciones del Instituto de Biología Aplicada, 6: 5-21. [9]

Margalef, R., 1958. Materiales para el estudio de las comunidades bióticas de las aguas dulces y salobres, principalmente delNE de España. Publicaciones del Instituto de Biología Aplicada, 28: 5-47. [10]
PARdo, L., 1944. Pescando en el estanque del Retiro. Calendario de Caza y Pesca, 14: 33-34. [11]

Velasco, J. L., 1978. Estudio limnológico del embalse de San Juan. Tesis Doctoral, Universidad Complutense de Madrid. 231 pp. [12]

Velasco, J. L., 1990. Lista faunística y bibliográfica de los Rotiferos (Rotatoria) de la Península Ibérica e Islas Baleares y Canarias. Asociación Española de Limnología, Publicación No 8. Madrid. 195 pp. [13]

Velasco, J. L., 1992. Limnología de la laguna de San Juan (Madrid): sucesión anual de las comunidades de rotíferos. Ecología, 6: 341-350. [14]

Velasco, J. L., Álvarez, M. \& Rubio, A., 1995. La laguna de gravera de El Campilo (Madrid): datos físico-químicos y biológicos. Ecología, 9:65-70. [15]

Velasco, J. L., Álvarez, M., Colomer, M. \& Rubio, A., 1996a. El «Mar de Ontígola» (Madrid): características limnológicas. Anales de Biología, 21(10): 93-104. [16]

Velasco, J. L., Álvarez, M. \& Rubio, A. 1996 b. Influencia de la ruptura de la termoclina sobre la comunidad de rotíferos plactónicos de una laguna meromíctica (Las Madres, Madrid). Ecología, 10: 523-532. [17]

Velasco, J. L. \& AvilÉs, J., 1998. Rotíferos del Parque Regional de la Cuenca Alta del Manzanares, Madrid: abundancia y distribución. Ecología, 12: 459-473. [18] 